\title{
An open real-time photoacoustic imaging scanner
}

\author{
Aneline DOLET \\ 1. Univ.Lyon, INSA-Lyon, Université \\ Claude Bernard Lyon 1, UJM-Saint \\ Etienne, CNRS, Inserm, CREATIS \\ UMR 5220, U1206, F-69621, LYON, \\ France \\ 2. Department of Information \\ Engineering, University of Florence, \\ Florence, Italy \\ aneline.dolet@creatis.insa-lyon.fr \\ Alessandro DALLAI \\ Department of Information \\ Engineering, University of Florence, \\ Florence, Italy \\ alessandro.dallai@unifi.it
}

\author{
Alessandro RAMALLI \\ 1. Lab. on Cardiovascular Imaging \& \\ Dynamics, Dept. of Cardiovascular \\ Sciences, KU Leuven, Leuven, Belgium \\ 2. Department of Information \\ Engineering, University of Florence, \\ Florence, Italy \\ alessandro.ramalli@kuleuven.be \\ Piero TORTOLI \\ Department of Information \\ Engineering, University of Florence, \\ Florence, Italy \\ piero.tortoli@unifi.it
}

François VARRAY

Univ.Lyon, INSA-Lyon, Université

Claude Bernard Lyon 1, UJM-Saint

Etienne, CNRS, Inserm, CREATIS

UMR 5220, U1206, F-69621, LYON, France

francois.varray@creatis.insa-lyon.fr

\author{
Enrico BONI \\ Department of Information \\ Engineering, University of Florence, \\ Florence, Italy \\ enrico.boni@unifi.it
}

\author{
Didier VRAY \\ Univ.Lyon, INSA-Lyon, Université \\ Claude Bernard Lyon 1, UJM-Saint \\ Etienne, CNRS, Inserm, CREATIS \\ UMR 5220, U1206, F-69621, LYON, \\ France \\ didier.vray@creatis.insa-lyon.fr
}

\begin{abstract}
Photoacoustic imaging is a functional technique based on the detection of acoustic waves originated from tissues excited by an optical source (laser pulses). When different laser wavelengths are used, this modality allows the discrimination of different media, which is promising for various medical applications. The acquisition system must show two main capabilities: 1) the real-time imaging of the region of interest and 2) the access to raw data to test reconstruction and processing methods under development. However, today's systems provide only one of these two requirements. Therefore, this study presents the development of a 2D hybrid ultrasound/photoacoustic real-time mode, based on the ultrasound open scanner ULA-OP256, which allows the access to raw data. The system validation was done on a $4 \%$ agar phantom containing two graphite mines illuminated by a pulsed laser at $1064 \mathrm{~nm}$. The test highlights the correct synchronization of the optical illumination with the photoacoustic acquisition, as well as the accurate display of photoacoustic and ultrasound images.
\end{abstract}

Keywords-real-time imaging, photoacoustic imaging, passive mode

\section{INTRODUCTION}

Multispectral photoacoustic imaging is an emerging medical technique to assess functional imaging of biological tissues by exploiting their wavelength-dependent optical absorption [1]. Pulsed laser excitations warm-up the optical absorbers. The temperature increase of optical absorbers leads to their thermal expansion that generates ultrasound waves. Ultrasound can finally back-propagate to the tissue surface where it is detected by the probe. The photoacoustic signal is then linked to the optical absorption of the imaged media [2]. In particular, the use of wavelengths in the range $600-900 \mathrm{~nm}$ allows distinguishing between oxygenated and deoxygenated blood, which is of interest for applications like the follow-up of diseases involving atheroma plaques [3] or carcinoma [4].

Nowadays, some of the commercial systems produce photoacoustic images in real-time but, unfortunately, provide limited access to raw data (e.g. Vevo LAZR, Visualsonics, Fujifilm) [5], or vice-versa (e.g. ULA-OP256, MSD Lab, Florence, Italy) [6]. However, both requirements are important for photoacoustic technology development: (1) the real-time operation allows following tissue or blood dynamics and (2) the raw data accessibility permit the development and/or the improvement innovative reconstruction and processing methods.

Therefore, this study reports on the development and validation of a continuous real-time photoacoustic imaging system based on the ULA-OP256 research scanner [7], which also allows full raw data accessibility. First, the materials and methods used in this study are presented (sec. II). 2D real-time imaging mode validation results are then shown (sec. III). Finally, the study conclusions and some perspectives on this new real-time photoacoustic imaging mode are given (sec. IV).

\section{MATERIALS AND METHOD}

\section{A. Optical excitation}

The multispectral optical excitation used in this study is a Nd:YAG pulsed laser (Quanta-Ray INDI Series, SpectraPhysics, USA), with pulse duration of $6 \mathrm{~ns}$ and a $10 \mathrm{~Hz}$ repetition rate. The laser is coupled with an optical parametric oscillator (OPO - versaScan, 
Spectra-Physics, USA) to allow the multispectral acquisitions. With this system, it is possible to acquire images with a wide excitation wavelength range, from $410 \mathrm{~nm}$ to $1250 \mathrm{~nm}$. In this study, the used wavelength was $1064 \mathrm{~nm}$ permitting a maximal optical energy of $200 \mathrm{~mJ}$ for each pulse.

\section{B. Ultrasound detection}

The ULA-OP256 scanner has been used in association with the linear array probe (model LA523E, Esaote S.p.A., Florence, Italy). The probe includes 192 elements; its center frequency is $7.8 \mathrm{MHz}$ with a bandwidth of $92 \%$ at $-6 \mathrm{~dB}$.

Active ultrasound images have been obtained with 32 plane wave transmissions ( 3 cycles, $8 \mathrm{MHz}$ frequency) using all the 192 elements of the probe. In active imaging mode, the echo signals received by all elements are used with a $8 \mathrm{MHz}$ demodulation frequency. In passive imaging mode, the photoacoustic images are reconstructed using only 128 elements and a $5 \mathrm{MHz}$ demodulation frequency.

\section{Optical excitation/ultrasound detection synchronization}

A photoacoustic image has to be reconstructed for each laser pulse, e.g. at $10 \mathrm{~Hz}$ frame rate, during the optical pulse illumination (6 ns, in blue in Fig. 1). The ULA-OP256 must be triggered by the laser to synchronise the photoacoustic acquisition with the optical excitation. However, since the scanner needs $14 \mu \mathrm{s}$ after the trigger signal arrival to effectively start the photoacoustic image, the laser pulse cannot be used to acquire a passive image during its own duration. Indeed, in this case, the photoacoustic image would be acquired $14 \mu \mathrm{s}$ after the optical pulse beginning, which means after the $6 \mathrm{~ns}$ optical excitation. At this instant, no photoacoustic signal is anymore generated; no passive signal can then be acquired. Hence, a custom electronic circuit was developed to synchronize the laser and the ULA-OP256. In particular, the $\mathrm{N}$-th laser pulse was used to trigger the next $(\mathrm{N}+1)$ photoacoustic acquisition. Therefore, the trigger signal needs to be delayed exactly by $99.986 \mathrm{~ms}$ from the previous pulse laser (trigger signal in red on Fig. 1, the yellow arrows highlight the trigger pulses for photoacoustic acquisitions). The delay needs to be accurately set to allow a well photoacoustic image reconstruction.
The available time between two consecutive laser pulses is exploited to acquire ultrasound B-Mode images. The trigger signal needs also to make these acquisitions possible. The red pulses without yellow arrows on Fig. 1 are used to this end.

\section{Real-time photacoustic mode}

The ULA-OP256 system has been programmed to produce interleaved plane wave (ultrasound) and passive (photoacoustic) B-Mode images. The multi-line parallel beamformer [8] of the scanner reconstructs one passive image consisting of 128 lines in $0.4 \mathrm{~ms}$ and one active image of 192 lines in $1 \mathrm{~ms}$. The two B-Mode images are superimposed on the screen to display a hybrid ultrasound/photoacoustic image in real-time.

\section{RESULTS}

The validation of the developed hybrid ultrasound/photoacoustic real-time mode has been done on the phantom shown in Fig. 2(a). It is a $4 \%$ agar phantom including two graphite mines. The yellow rectangle on Fig. 2(a) highlights the ultrasound region of interest while the orange ellipse highlights the laser beam size which corresponds to the area generating photoacoustic signals. The phantom is excited by the laser beam at $1064 \mathrm{~nm}$ (Fig. 2(b), the visible green light is due to a residual laser component at $532 \mathrm{~nm}$ ). The phantom was translated from left to right by maintaining the plan of the graphite mines aligned with the imaged region. The photoacoustic signal is in blue while the active B-mode image is in grayscale (Fig. 2(c)).

Some artefacts are visible in Fig. 2(c) particularly at the second phantom position (t1), highlighted by white arrows. They are probably due to the photoacoustic signal multiple echoes. Indeed, both mines refract a part of the ultrasound waves which are finally received by the ultrasound probe after some echoes. Also, since the ultrasound waves do not pass well through the graphite mines, the photoacoustic signal of the bottom graphite mine is lower than that of the upper one (Fig. 2(c)-t1).

Fig. 2(c) however shows the accurate synchronization of the optical excitation with the photoacoustic acquisition. Indeed, the photoacoustic signal is well reconstructed at a good location. It can also be seen that the passive and active

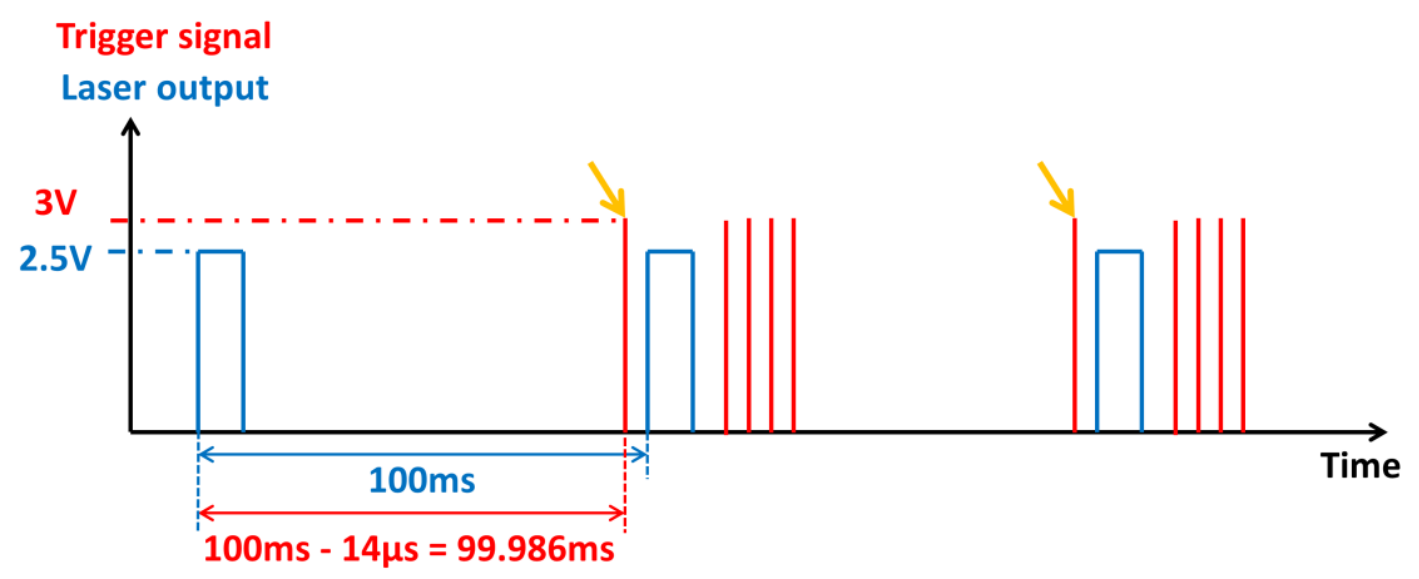

Fig. 1. Scheme of the ULA-OP256 triggereg by the lasser pulse. The red pulses with yellow arrows represent the trigger for photoacoustic acquisitions while the other red pulses are for plane wave B-Mode acquisitions. 


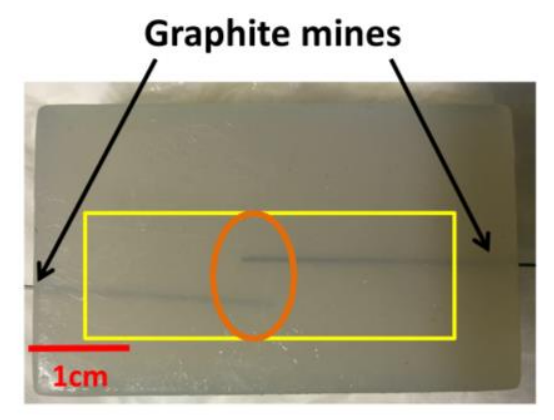

(a)

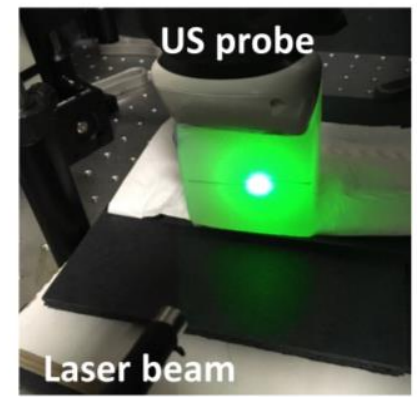

(b)
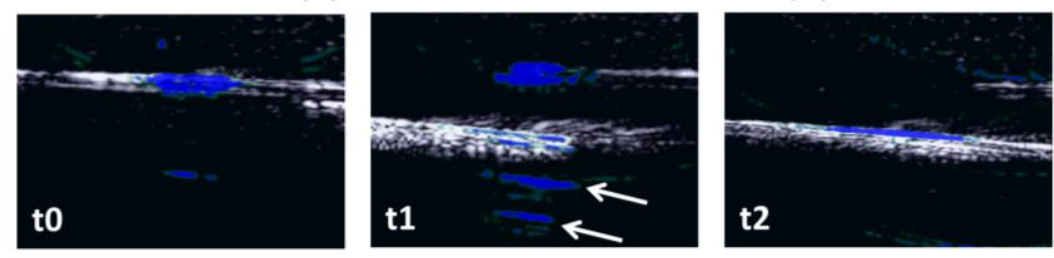

(c)

Fig. 2. (a) Cross-sectional phantom image: the yellow rectangle highlights the region imaged with the active mode; the orange ellipse, the laser beam size and the black arrows, both graphite mines. (b) Photoacoustic set-up. The visible green light is due to a residual laser component at 532 nm. (c) Screenshots of the real-time display for different phantom positions. The photoacoustic signal is in blue. The white arrows highlight some multiple echoes artefacts.

images are correctly displayed with fair superimposition of both signals in real-time. Finally, it may be reminded that the ULA-OP256 scanner system allows the accessibility to both active and passive raw data which is interesting for off line post-processing and to test reconstruction or processing methods under development.

\section{CONCLUSION AND DISCUSSIONS}

The synchronization of an external laser source with ULA-OP256 allowed exploiting the parallel beamforming capability of such system to continuously produce photoacoustic images at $10 \mathrm{~Hz}$. The sample frames in Fig. 2(c), extracted from the real-time screen capture, highlight the correct synchronization of the optical illumination and passive ultrasound acquisition, as well as the accurate display of passive and active images. In addition to the continuous real-time display modality, ULA-OP256 makes also all the raw data available for possible off line processing including quantification method improvements, which are currently under development.

A similar mode has also been developed for 3D imaging using a matrix array probe of $8 \times 24$ elements (Vermon, France); its center frequency is $3.84 \mathrm{MHz}$ with a bandwidth of $65.1 \%$ at $-6 d B$. Diverging waves are used for the ultrasound transmission of active images. Further studies are now done to finalize the validation of this 3D development with accurate synchronization and real-time display.

\section{ACKNOWLEDGMENT}

This work was supported by the LABEX CELYA (ANR-10-LABX-0060) and LABEX PRIMES (ANR-10LABX-0063) of Université de Lyon, within the program "Investissements d'Avenir" (ANR-11-IDEX-0007) operated by the French National Research Agency (ANR). It was also performed within the framework of the Universite FrancoItalienne (VINCI 2016) and the Région Rhône-Alpes (EXPLORA DOC). The authors also would like to thank the research council of Université Claude Bernard Lyon 1 for his financial support and the LISA-CEA-LETI for providing part of the laser system equipment.

\section{REFERENCES}

[1] M. Vallet, F. Varray, J. Boutet, J.M. Dinten, G. Caliano, A.S. Savoia, and D. Vray, "Quantitative comparison of PZT and CMUT probes for photoacoustic imaging: experimental validation," Photoacoustics 8, pp.48-58, 2017.

[2] P. Beard, "Biomedical photoacoustic imaging", Interface Focus 1, pp.602-631, 2011

[3] K. Jansen, M. Wu, A.F.W. van der Steen, and G. van Soest, "Lipid detection in atherosclerotic human coronaries by spectroscopic intravascular photoacousic imaging", Optics Express, vol. 21(18), pp.21472-21484, 2013.

[4] D.R. Bauer, R. Olafsson, L.G. Montilla, and R.S. Witte, "In vivo multi-modality photoacoustic and pulse echo tracking of prostate tumor growth using a window chamber," in $\mathrm{BiOS}$. International Society for Optics and Photonics, 2010, pp.75643B-75643B.

[5] C.J. Arthuis, A. Novell, F. Raes, J.M. Escoffre, S. Lerondel, A. Le Pape, A. Bouakaz, and F. Perrotin, "Real-time monitoring of placental oxygenation during maternal hypoxia and hyperoxygenation using photoacoustic imaging", PLOS ONE, vol. 12(1), pp.01698500169850-11, January 2017.

[6] E. Boni, A. Yu, F. Freear, J. Jensen and P. Tortoli, "Ultrasound Open Platforms for Next-Generation Imaging Technique Development", IEEE Transactions on Ultrasonics Ferroelectrics and Frequency Control, 65:7, pp. 1078-1092, 2018.

[7] E. Boni, L. Bassi, A. Dallai, F. Guidi, V. Meacci, A. Ramalli, S. Ricci and P. Tortoli, "ULA-OP 256: A 256-channel open scanner for development and real-time implementation of new ultrasound methods", IEEE Transactions on Ultrasonics Ferroelectrics and Frequency Control, 63:10, pp.1488-1495, 2016.

[8] E. Boni, L. Bassi, A. Dallai, V. Meacci, A. Ramalli, M. Scaringella, F. Guidi, S. Ricci and P. Tortoli, "Architecture of an Ultrasound System for Continuous Real-time High Frame Rate Imaging”, IEEE Transactions on Ultrasonics Ferroelectrics and Frequency Control, Volume 64, pp. 1276-1284, 2017. 